\title{
Complications of Massive Blood Transfusion
}

\author{
Authors \\ Ketan Vagholkar ${ }^{1}$, Madhavan Iyengar ${ }^{2}$, Suvarna Vagholkar $^{3}$, Inder Maurya ${ }^{4}$ \\ ${ }^{1}$ Professor, ${ }^{2}$ Associate Professor, ${ }^{3}$ Research Assistant, ${ }^{4}$ Senior Resident \\ Department of Surgery, D.Y.Patil University School of Medicine, Nerul, Navi Mumbai-400706 MS. India \\ Corresponding Author \\ Dr. Ketan Vagholkar \\ Annapurna Niwas, 229 Ghantali Road Thane 400602 MS. India \\ Email: kvagholkar@yahoo.com, Mobile: 9821341290

\begin{abstract}
Blood transfusion is one of the commonest accompaniments of surgical practice. With a growing incidence of major polytrauma by vehicular accidents, bomb blasts and fires, awareness of the basic concepts underlying massive blood transfusion practice with special reference to the complications is essential. The paper outlines the pathophysiologic mechanisms underlying the various complications of massive blood transfusion.

Key Words: Massive blood transfusion complications.
\end{abstract}

\section{Introduction}

Massive Blood Transfusion (MBT) is an integral part of surgical practice. Advances in blood banking technology have given a great impetus to the surgeons, enabling them to undertake complex surgical procedures. However management of trauma patents continues to pose the greatest challenge to the attending surgeon. Blood Transfusion practice ranging from transfusion of isolated units of blood to massive transfusion has enabled attending surgeons to save innumerable lives. However it is imperative for the surgeons to be aware of the complications of massive blood transfusion which can not only increase the morbidity but can also lead to mortality.

Massive transfusion is defined as replacement of patients total blood volume within 24 hours. ${ }^{[1]}$ Since blood happens to be the integral component of the body's homeostatic system, the sequel to massive transfusion can lead to disastrous consequences.

A massive blood transfusion can cause gross changes in the body biochemistry, coagulopathy and hypothermia. These three deleterious effects are interlinked and propagate each other causing a complete collapse of the body homeostasis as well as weakening of the metabolic response to trauma.

Biochemical changes: A series of diverse biochemical changes accompany massive blood transfusion. ${ }^{[2,3,4,5]}$

Hypocalcemia: RBC transfusions contain only traces of citrates whereas platelets and FFP transfusions contain higher concentration of citrate. A standard blood transfusion may hardly cause any alteration in the calcium levels, as the first pass of citrate through the liver will 
automatically lead to metabolic breakdown in less than 5 minutes.

However when the number of units transfused is higher accompanied with hypothermia then the liver function gets altered thereby decreasing the clearance of citrate from the blood leading to increased concentration of citrate in the circulation. This causes binding of citrate to ionic calcium leading to hypocalcemia. Clinically it can manifest in the form of tetany, prolonged QT interval, decreased myocardial contractility, hypotension, narrow pulse pressure and elevated central venous pressure. Hypocalcemia may also predispose to hyperkalemia induced arrhythmia as well as ventricular fibrillation and pulseless electrical activity. Hence monitoring of calcium levels is mandatory accompanied by periodic supplementation with intravenous calcium gluconate to maintain the levels of ionic calcium within the normal range.

Hypomagnesaemia is another accompaniment of massive transfusion. This may be due to two reasons that is overzealous administration of magnesium poor fluids, as well as binding of magnesium to citrates. As a result, citrate toxicity leads to concomitant hypocalcaemia and hypomagnesaemia. Low levels of magnesium can lead to prolonged QT interval, arrhythmia as well as contribute to coagulopathy.

Hyperkalemia is one of the dreaded complications of massive blood transfusion. The extracellular potassium increases in stored blood which is attributable to the inactivation of the RBC membrane ATPase pump. The potassium levels in the blood increase with time. With seven days of storage the concentration may be $12 \mathrm{meq} /$ liter and may increase to $32 \mathrm{meq} / \mathrm{liter}$ after 21 days. As soon as the unit is transfused the potassium is taken up by the RBC as the functioning of the ATPase pump is restored. Hence in a small volume transfusion, hyperkalemia is a transient phenomenon devoid of any toxic effect. However during massive blood transfusion wherein large volumes of blood are given through central lines, a large bolus of extra cellular potassium may reach the right heart prior to intra cellular uptake leading to dysrhythmias which may be compounded by hypocalcemia. The best way to reduce the deleterious effects of hyperkalemia is to transfuse the blood through anintravenous line which is far away from the right atrium or alternatively by using washed RBC's. Concomitant correction of acidosis is important in enhancing the intra cellular uptake of potassium. However if the level continues to remain high glucose insulin drips, beta 2 agonist, bicarbonates and intra venous calcium may be indicated.

Storage of blood for longer periods of time decreases the ability of transfused cells to deliver the oxygen to the tissues. This is because RBC's cannot make their way through the micro circulation. In addition the concentrations of 2-3 DPG decreases in stored RBC's thereby preventing the uploading of oxygen at tissue levels. Therefore in the context of MBT the older units of RBC's will be unable to deliver adequate oxygen to hypo perfuse tissues.

Coagulopathy: Coagulopathy in polytrauma patents may have a twofold etiology. Acute traumatic coagulopathy by virtue of acidosis, hypothermia and dilutional coagulopathy. ${ }^{[6,7]}$ This is compounded by massive transfusion with its own inherent mechanisms. During trauma related hemorrhage there is an increased lactate production in hypo perfused tissues. This compounded by the citrate phosphate dextrose adenine solution in stored RBC units. Once transfused the acid present in the blood products is metabolized in the liver. However due to grossly altered liver metabolism there is worsening of acidosis with consequences such as dysrhythmias, decreased cardiac contractility, hypotension and decreased response to catecholamine. Acidosis can by itself weaken the coagulation pathway by decreasing the enzymatic activity of the clotting factors, reduced thrombin production and impaired platelet aggregation. The toxic effects of acidosis can be reduced by using fresh blood 
accompanied by administering alkaline solutions like bicarbonates.

Hypothermia: Hypothermia is defined as core temperature less than 36 degrees Centigrade. ${ }^{[4,}$ ${ }^{8]}$ This is a common accompaniment of trauma patients. Hypothermia by itself may be due to the environmental conditions at the time of injury, during evacuation and during exposure for evaluation or surgery. Initial infusion of a large amount of inadequately warmed crystalloid solution may induce hypothermia. This followed by a MBT in temperature range of 1-6 degree Centigrade will worsen the hypothermia. Hypothermia can cause deleterious effects on the haemostatic mechanisms, impairment of oxygen delivery by the RBC's, decreased cardiac output, increased risk of cardiac arrhythmias, increased cardiac toxicity secondary to electrolyte derangement and gross alterations of platelet functions thereby distorting the coagulation cascade predisposing to DIC.DIC may also be caused by virtue of dilutional thrombocytopenia associated with massive transfusion. Low platelet content, deficient labile clotting factors 5and 8 may compound the coagulopathy. The administration of colloids such as hydroxyl ethyl starch can adversely affect the function of Von Willebr and factor. Hence it is advisable to limit the administration of colloids as far as possible. Carrying out conventional coagulation tests may not always be possible during the course of MBT. Hence newer testing like real time TEG may help to assess patent's ongoing coagulation status serving as guide for further transfusion.

Immunological complications are often the fall out of MBT. ${ }^{[8,9,10]}$ Acute hemolytic reactions are seen in patients who have been administered uncrossed type $\mathrm{O}$ blood and latter receive type specific blood. This is most probably due to the transfused isoaglutinin from whole blood reacting against type $A \& B$ antigens present in type specific blood. Microchemerism which is harboring of small amount of cells that originated in genetically different individuals may also give rise to immunological complications.
Immunomodulation is an immune based deleterious effect which is taken advantage of in transplant patents. It predisposes to increased risks of infection, acute lung injury, ARDS, and multi organ failure.

Transfusion induced acute lung injury (TRALI) occurs within 6 hours of transfusion, but could be delayed up to even 24 hours post transfusion. ${ }^{[8,11]}$ The type of blood product determines the risk of TRALI which could be 1/5000 units of PRBC's, 1/2000units of FFP's, $1 / 400$ units of platelets. Clinically it leads to the development of non-cardiogenic pulmonary edema, severe hypotension and bilateral fluffy in filtrates in the lungs.

It is caused by an immune mediated process where in the donor antibodies activate the receptors on the leucocytes causing pulmonary injury, endothelial damage and capillary leakage. It has a high mortality of $25 \%$.

Treatment comprises of supportive care in form of mechanical ventilation, fluids and inotropes. ${ }^{[12]}$ This condition needs to be differentiated from transfusion associated circulatory overload which is accompanied with hydrostatic pulmonary edema which occurs in $1 \%$ of transfusion reactions.

\section{Conclusion}

Massive blood transfusion is pivotal in saving patients who are exsanguinating due to polytrauma. However the complications of MBT are as lethal and complex. Awareness of the pathophysiology, diagnosis and management is essential for the attending surgeon.

\section{Acknowledgements}

We would like to thank Mr.Parth K. Vagholkar for his help in typesetting the manuscript.

\section{References}

1. Donaldson MDJ, Seaman MJ, Park GR. Massive Blood Transfusion. Br J Anaesth 1992; 69: 621-30. 
2. Bux J. Transfusion related acute lung injury (TRALI): a serious adverse event of blood transfusion. Vox Sang. 2005; 89:110.

3. Kopko PM, Holland PV. Mechanisms of severe transfusion reactions. TransfusClin Biol. 2001;8: 278-81.

4. Kirkley SA. Proposed mechanism of transfusion induced immunomodulation. ClinDiagn Lab Immunol. 1999; 6: 652-57.

5. Sihler KC, Napolitano LM. Complications of massive transfusion. Chest. 2010; 137: 209-220.

6. Henderickson JE, Hillyer CD. Noninfectious srious hazards of transfusion. Anaesth Analg. 2009; 108: 759-769.

7. Miller RD. Massive blood transfusion: The impact of Vietnam military data on modern civilian transfusion medicine. Anaesthesiology. 2009; 110: 1412-1416.

8. Teague G, Gaylard D. Transfusion related lung injury. Anaesth Intensive Care. 2005; 33: 124-127.

9. Spinella PC. Warm fresh whole blood transfusion for severe haemorrhage: US military and potential civilian applications. Crit Care Med. 2008; 36 (supp17): 340345.

10. Brohi K, Singh J, Heron M, Coats T. Acute traumatic coagulopathy. J Trauma. 2003; 54: 1127-30.

11. Meibner A, Achlenke P. Massive Bleeding and Massive Transfusion. Transfus Med Hemother. 2012; 39: 73-84.

12. Dutton RP. Resuscitative strategies to maintain homeostasis during damage control surgery. Br J Surg. 2012; 99(Suppl1): 21-8. 